\title{
State Estimation in Two Time Scales for Smart Distribution Systems
}

\author{
Antonio Gómez-Expósito, Fellow, IEEE, Catalina Gómez-Quiles, Member, IEEE, \\ and Izudin Džafić, Senior Member, IEEE
}

\begin{abstract}
The monitoring of distribution systems relies on a critical set of pseudomeasurements and a varying but low number of redundant measurements. In the light of the different refreshing rates of both types of information, this paper considers a state estimation model structured in two time scales. Possibilities and limitations of the proposed model are discussed, and illustrated on a real distribution system comprising a diversity of load patterns.
\end{abstract}

Index Terms-Smart distribution system, state estimation, twotime scale measurements.

\section{INTRODUCTION}

$\mathbf{T}$ HE NOTION of state estimation (SE) for transmission systems can be traced back to the seventies [1]. Some twenty years later, SE algorithms specifically tailored to distribution systems were introduced [2], [3]. In practice, however, it has not been until very recently that SE tools for distribution feeders have been comprehensively considered [4]-[6].

Smart grid developments are progressively bringing more and more information to distribution management systems (DMS), allowing applications that were long ago conceptually mature but still waiting for the required infrastructure to be deployed at the distribution level [7], [8]. Eventually, the massively distributed nature of medium-voltage and lowvoltage subsystems, and the resulting communication bottlenecks, will force utilities to consider some kind of hierarchical organization in today's fully centralized DMS [9]. Indeed, only if raw data are processed in a local manner [10] will it be possible for new and ubiquitous sources of information, such as smart meters and the associated concentrators, to be scanned at rates which are fast enough for real-time network operation.

Until this partly decentralized environment arrives, DMS operators can only expect to have once-a-day or fewtimes-a-day values of energy consumed by customers connected to the distribution system [11]. This has motivated the development of heuristic methods combining load flow calculations [13], [14], machine learning functions [12] or

Manuscript received December 23, 2013; revised April 18, 2014; accepted June 29, 2014. This work was supported by Siemens and the Andalusian Government under Grant TEP-07411 [Cambiadores de Tomas Estáticos para Transformadores de Potencia (CATEST) Project]. Paper no. TSG-00932-2013.

A. Gómez-Expósito and C. Gómez-Quiles are with the Department of Electrical Engineering, University of Seville, Sevilla 41092, Spain (e-mail: age@us.es; catalinagq@us.es).

I. Džafić is with Siemens AG, D-90459 Nuremberg, Germany (e-mail: idzafic@ieee.org).

Color versions of one or more of the figures in this paper are available online at http://ieeexplore.ieee.org.

Digital Object Identifier 10.1109/TSG.2014.2335611 pattern-based load allocation [15] with ad hoc SE techniques. What these hybrid schemes generally have in common is a preprocessing phase in which delayed smart meter data or daily load patterns are somehow exploited to generate pseudomeasurements for the SE phase.

In the foreseeable future, if not in the near term, smart meter data will be collected and preprocessed by substationlevel management systems, at much faster scan rates than those achievable if every piece of information had to be gathered at the centralized DMS. Whereas a DMS is in charge of an entire system, typically serving several million customers, a 60-MW primary substation may serve three orders of magnitude less customers, whose smart meter data are in turn concentrated at less than a hundred intermediate points [generally secondary substations serving the low voltage (LV) subsystem]. Having these data collected at the primary substation, at rates ranging from 5 to 20 times an hour, is a feasible choice even with today's bandwidths and technology.

In this context, the substation-level SE tool will have to deal with two heterogeneous types of information, as explained in more detail in the next section: 1) regular supervisory control and data acquisition (SCADA) measurements, and eventually those coming from new smart grid sensors, captured every few seconds; and 2) smart meter (or smart meter concentrator) readings and distributed generation production, updated every few minutes.

This naturally leads to an information processing model in two time scales. Even though two-time-scale problems have long been known and exploited in several engineering fields (see for instance [16]-[19]), including SE of chemical or biological processes [20], to the authors knowledge such a notion has not been explored so far in power system SE. This paper is aimed at developing and testing a weighted least-squares (WLS) SE for distribution feeders with measurements captured in two time scales. A thorough analysis performed in [21] concluded that WLS SE is the most appropriate estimator for the low redundancy levels typically found in distribution systems (hardly higher than 1.1 even when pseudomeasurements are included). This work is inspired by the recent development of the so-called state reconstruction technique [22], by which few fast-rate phasor measurement units (PMUs) are used to reconstruct intermediate states between consecutive executions of slow-rate conventional SE.

The structure of this paper is as follows. Section II reviews and classifies the information sources available in smart distribution systems. Then, Section III presents the SE model in the 
presence of two-time-scale measurements. Next, Section IV discusses the limitations of the resulting SE model when low measurement redundancy and pseudomeasurement obsolescence is considered. Simple interpolation and extrapolation mechanisms, usually improving the accuracy of the estimates, are considered in Section V. Finally, the proposed models and solution refinements are tested on a real distribution system, where their theoretically expected behavior is confirmed.

\section{SOURCES OF INFORMATION IN SMART DISTRIBUTION SYSTEMS}

Unlike transmission and subtransmission systems, where real-time telemetry provides sufficient redundancy to assure network observability, medium voltage (MV) distribution feeders have so far lacked the required infrastructure (sensors and telecommunication) allowing the operating point to be accurately determined.

In the upcoming smart grid paradigm, though, distribution systems will have to cope with an heterogeneous set of information sources, most of them not yet available at the DMS, which can be roughly classified into the following categories.

1) Remote terminal unit (RTU) measurements captured at HV-MV substations, collected by the SCADA system of the DMS at rates ranging from few seconds to about a minute (in general, much lower refreshing rates than those employed at transmission-level SCADAs [23]). As far as radial feeders are concerned, such measurements typically reduce to the MV busbar voltage magnitude and head line currents (assuming passive loads with an average power factor, this allows the total P\&Q delivered by the feeder to be computed). So far, this is essentially the only telemetered information at the MV level for a majority of utilities which, unless a fault occurs, can only have a very crude idea of what is going on downstream with the help of load allocation techniques (item 4).

2) Higher reliability standards [i.e., lower system average interruption frequency index/system average interruption duration index (SAIFI/SAIDI) indexes] are forcing distribution utilities to deploy more and more feeder automation devices, including remotely-operated intermediate switching points for fault management. Once the required communication channel is available, such points can be converted into true RTUs with very little extra investment. In fact, most vendors currently offer this product in their catalogs. The information provided by these additional RTUs can be useful for both fault location and state estimation purposes.

3) Distributed generation is already a reality and will increasingly spread in many radial feeders worldwide. Depending on the specific regulation and rated power, the production of distributed generators (DGs) is required to be monitored at different rates, ranging from day-ahead hourly forecasting to real telemetry periodically submitted to the DMS.

4) Distribution utilities have customarily kept a more or less elaborated data base of historic load patterns/profiles. This information originates in several sources, including load forecasting, load allocation techniques in combination with feeder head measurements, characteristic power factor values of aggregated loads and systematic metering campaigns performed at specific points. The feeder-level state estimator can benefit from these not very precise values of $P$ and $Q$, which can be used as pseudo-measurements to extend the observable area.

5) The latest and eventually most important addition to the list of information sources at the feeder level comes from the automatic meter reading/advanced metering infrastructure (AMR/AMI) infrastructure (typically smart meter concentrators), provided the right communication bridge is built between AMI and DMS subsystems. Nowadays this information is collected once a day in many systems but, depending on bandwidth availability, snapshot latencies of up to $15 \mathrm{~min}$ have been reported.

Notice that not all of the above data will necessarily reach the DMS, but may remain at an intermediate place much closer to the points where they are captured from the field. In the hierarchical control system architecture envisioned elsewhere [7], [9], [10], the right place where the raw information should be collected and processed is the distribution substation, since there are currently no technical barriers for a state estimator to be implemented in this environment.

For the purposes of this paper, all sources of information summarized above, to which the feeder-level state estimator can resort, will be grouped in two broad classes of different nature, each with different accuracy and latency.

1) Telemetered data provided by RTUs (items 1, 2, and in some cases, 3). This comprises quite accurate snapshots captured with latencies ranging from few seconds to about a minute. The set of measurements is insufficient in any case to assure network observability.

2) Pseudomeasurements (items 3, 4, and 5). Updated at intervals ranging from $15 \mathrm{~min}$ to $24 \mathrm{~h}$, these bus-level data are barely critical for observability purposes.

Clearly, in order to achieve a minimum redundancy level, both information types should be properly combined, which leads to the particular SE model described in the sequel.

\section{State Estimation in Two Time Scales}

Let $z_{m}$ and $z_{p}$ denote the fast-rate measurement and slowrate pseudomeasurement vectors, respectively. As suggested by Fig. $1, z_{m}$ snapshots are updated at regular intervals of width $T_{m}$, while $z_{p}$ is refreshed at much wider intervals of period $T_{p}=n T_{m}$. Between two consecutive snapshots of $z_{p}$, $n$ snapshots of $z_{m}$ are captured ( $n=4$ in the figure).

At a given time instant, $t_{k}$, the available information is composed of the current snapshot $z_{m, k}$ and the past pseudomeasurement value $z_{p, j}$. Therefore, the faster the load increases or decreases the quicker and more obsolete $z_{p, j}$ becomes. When the sign of the slope does not change between $t_{j}$ and $t_{j+n}$, the worst condition in terms of pseudomeasurement obsolescence arises for $t_{j+n-1}$, just before $z_{p}$ is updated again.

Dropping for simplicity the discrete-time indices, the resulting measurement model is

$$
\left[\begin{array}{c}
z_{p} \\
z_{m}
\end{array}\right]=\left[\begin{array}{c}
h_{p}(x) \\
h_{m}(x)
\end{array}\right]+\left[\begin{array}{c}
\varepsilon_{p} \\
\varepsilon_{m}
\end{array}\right]
$$




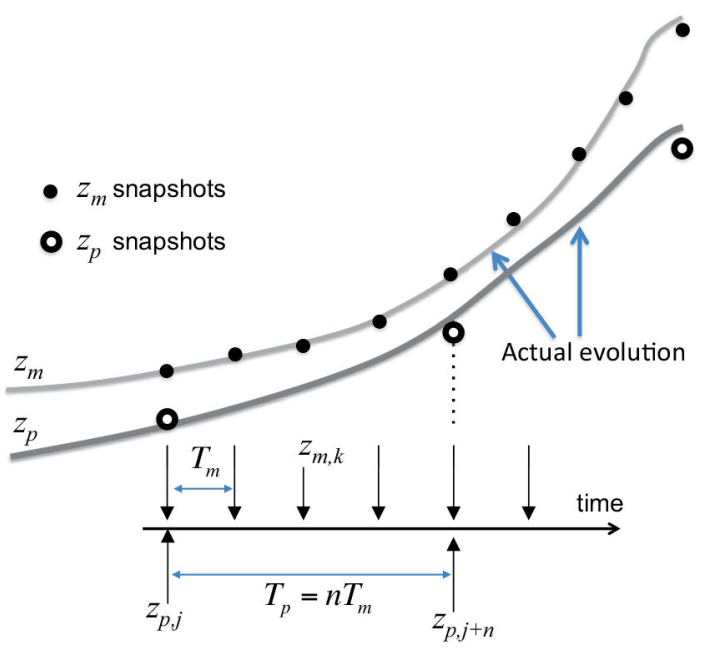

Fig. 1. Measurement (fast) and pseudomeasurement (slow) snapshots of two electrical quantities.

where $h_{p}(\cdot)$ and $h_{m}(\cdot)$ represent the respective measurement functions and $\varepsilon_{p}$ and $\varepsilon_{m}$ the associated errors.

The WLS SE solution is obtained by iteratively solving the normal equations

$$
\begin{aligned}
\left(H_{p}^{T} W_{p} H_{p}+H_{m}^{T} W_{m} H_{m}\right) \Delta x= & H_{p}^{T} W_{p}\left[z_{p}-h_{p}(x)\right] \\
& +H_{m}^{T} W_{m}\left[z_{m}-h_{m}(x)\right]
\end{aligned}
$$

where the weighting coefficients should reflect whenever possible the information uncertainty. If errors can be assumed to be Gaussian, the maximum likelihood estimation is obtained when

$$
W_{p}^{-1}=\operatorname{cov}\left(\varepsilon_{p}\right) ; \quad W_{m}^{-1}=\operatorname{cov}\left(\varepsilon_{m}\right) .
$$

$\operatorname{cov}(\cdot)$ represents the error covariance matrix. Notice that the uncertainty of $\varepsilon_{p}$ is generally much higher than that of $\varepsilon_{m}$.

The special structure of the normal (2) can be exploited to save computational effort. Considering the relatively few number of measurements in vector $z_{m}$, a major source of computational saving arises when the Cholesky factorization of the gain matrix is not repeated at each SE run, but only when the set $z_{p}$ is updated. Approximating the gain matrix in this fashion may slightly increase the number of iterations, particularly when loads evolve quickly, but will not affect the solution as long as the right-hand side of (2) is exactly computed. Needless to say, using the solution of the previous run as starting point, rather than the customary flat start profile, is a convenient strategy to save iterations.

\section{Limitations Arising From the Use of ReducED REDUNDANCY LEVELS}

This section is devoted to qualitatively analyzing the limitations of the two-scale state estimator (TSSE) in a context characterized by extremely low redundancy levels. Intuitively, one expects that adding a few branch Ampere measurements, scattered throughout the feeder, to the set of bus pseudomeasurements, will always improve the estimate of relevant quantities (bus voltage magnitudes and branch power flows), which is true so long as the feeder is taken as a whole.

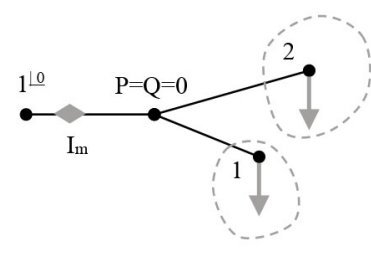

(a) (b)

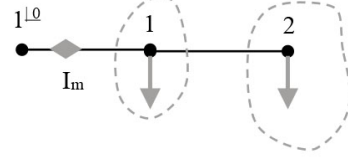

Fig. 2. Generic scenarios illustrating an Ampere-measured branch with two pseudo-measured buses downstream. (a) Two major laterals, each with an equivalent load. (b) A single lateral load plus the remaining aggregated load.

However, depending on whether or not all loads downstream have coincident evolution patterns, branch current measurements may or may not be helpful to improve the estimates of certain individual quantities when pseudomeasurements are not duly updated.

As explained below, this limitation stems from the combination of two adverse factors: 1) low redundancy of RTU measurements, clearly insufficient to render the network observable; and 2) gradual obsolescence of barely critical pseudomeasurements as time elapses, of particular relevance in periods when bus injections change at a fast rate.

In order to illustrate the analysis it is sufficient to consider the two simplified radial feeders shown in Fig. 2. In both cases, in addition to the head bus voltage, there is an Ampere measurement at the branch which is closer to the feeder head, while only $P \& Q$ pseudomeasurements are available at the two buses downstream. Notice that, in spite of their simplicity, such reduced feeders can be representative, in equivalent form, of different realistic situations, by simply playing with the relative sizes of $P_{1}-Q_{1}$ and $P_{2}-Q_{2}$. For instance, in case (a) each bus may represent the aggregated load of two main laterals downstream a bifurcation, both of similar size. In case (b), bus 1 can be a single load while bus 2 may represent the aggregated load of the remaining buses downstream, etc.

Instead of using an exact SE model, the same qualitative conclusions can be reached, with much less elaborated algebra, by adopting a lossless model with flat voltage profile in which active and reactive power injections constitute the state variables.

Given the latest pseudomeasurement values $P_{1}^{m}-Q_{1}^{m}$ and $P_{2}^{m}-Q_{2}^{m}$ and the most recent current measurement, $I_{m}$, which is assumed to be much more accurate than power pseudomeasurements, the WLS estimates for $P_{1}, P_{2}, Q_{1}$, and $Q_{2}$ can be analytically obtained. As shown in the Appendix, the active power estimates are

$$
\begin{aligned}
& \hat{P}_{1}=P_{1}^{m}+\Delta P_{1} \\
& \hat{P}_{2}=P_{2}^{m}+\Delta P_{2}
\end{aligned}
$$

where

$$
\begin{aligned}
\Delta P_{1} & =\frac{w_{2}}{w_{1}+w_{2}}\left(K_{m}-1\right)\left(P_{1}^{m}+P_{2}^{m}\right) \\
\Delta P_{2} & =\frac{w_{1}}{w_{1}+w_{2}}\left(K_{m}-1\right)\left(P_{1}^{m}+P_{2}^{m}\right) .
\end{aligned}
$$

$w_{1}$ and $w_{2}$ represent the weights of $P_{1}^{m}$ and $P_{2}^{m}$, respectively, and $K_{m}$ is the ratio

$$
K_{m}=\frac{I_{m}}{\sqrt{\left(P_{1}^{m}+P_{2}^{m}\right)^{2}+\left(Q_{1}^{m}+Q_{2}^{m}\right)^{2}}} .
$$


Notice that $K_{m}>1$ if the total load downstream has increased since slow-rate pseudomeasurements were updated, which is reflected in higher values of more recent $I_{m}$ snapshots, while $K_{m}<1$ when the total load has decreased. Similar expressions are obtained for reactive powers by simply replacing $P$ with $Q$ (for this reason, only active powers will be paid attention to in the sequel).

In low-redundancy scenarios, like those considered in this paper, the influence of the weighting coefficients $w_{1}$ and $w_{2}$ in the WLS estimates is crucial. In practice this poses a major problem, since knowing at each time instant the real uncertainty of pseudomeasurements is far from trivial. In this regard, it is worth considering the following two cases.

1) Same weights adopted $\left(w_{1}=w_{2}\right)$. This would lead to

$$
\Delta P_{1}=\Delta P_{2}=\left(K_{m}-1\right) \frac{P_{1}^{m}+P_{2}^{m}}{2}
$$

which means that both $P_{1}^{m}$ and $P_{2}^{m}$ will be corrected by the same amount to yield the respective estimates. In absence of any other information this will be acceptable provided both loads are of similar size. However, assume for instance that $P_{1} \gg P_{2}$. Then, according to (6), even small changes in $P_{1}$ might lead to relatively high deviations in the smaller load, $P_{2}$, irrespective of whether this load has really changed or not.

2) Weights inversely proportional to the pseudomeasurement $\left(w_{i}=1 / P_{i}^{m}\right)$. In this case, it is easy to see that

$$
\Delta P_{i}=\left(K_{m}-1\right) P_{i}^{m} \quad \Rightarrow \quad \hat{P}_{i}=K_{m} P_{i}^{m} \quad(i=1,2)
$$

which means that each power will be corrected in proportion to its size. In other words, for low-redundancy scenarios, like the simplified ones represented in Fig. 2, the total load variation detected by an Ampere measurement at a given feeder section is prorated among the loads located downstream in proportion to their respective sizes.

Note that, irrespective of the weights adopted, the signs of both $\Delta P_{1}$ and $\Delta P_{2}$ will be the same, according to (4), as determined by the value of $K_{m}$. If the total load increases (decreases) then $K_{m}>1\left(K_{m}<1\right)$ and both estimates, $\hat{P}_{1}$ and $\hat{P}_{2}$, will be higher (lower) than the outdated pseudomeasurements, $P_{1}^{m}$ and $P_{2}^{m}$. Indeed, this is an expected result for the low redundancy considered, since there is no way to know whether both loads have actually increased (decreased) or not (it is worth stressing that replacing $I_{m}$ by power flow measurements is not helpful in this regard).

In feeder sections where all transformer loads downstream of an Ampere measurement evolve in an homogeneous way, which happens when most customers have similar patterns, this may not be a real limitation. However, in feeders comprising a mix of customers (residential, industrial, municipal, etc.) some transformer loads may be increasing while others are simultaneously decreasing, and combining few Ampere measurements with critical pseudomeasurements can be counterproductive, particularly if sudden load changes take place.

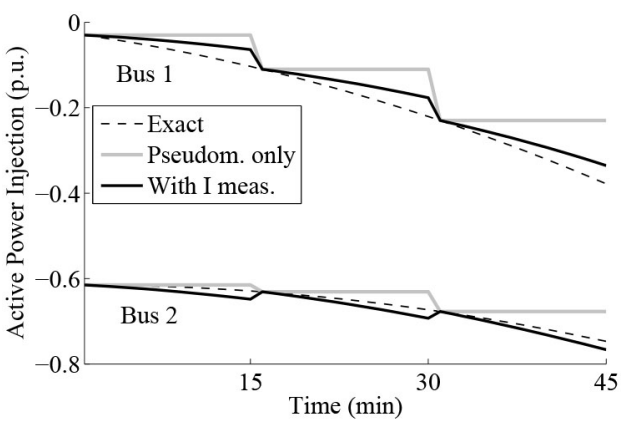

Fig. 3. Tutorial example with homogeneous load trends.

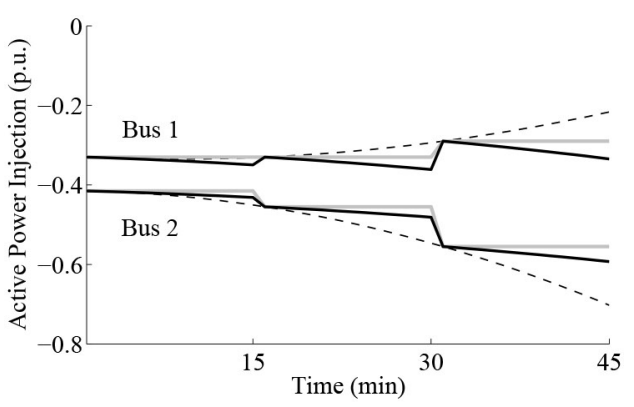

Fig. 4. Tutorial example with opposite load trends.

Fig. 3 illustrates a case in which both $P_{1}$ and $P_{2}$ decrease during the next $45 \mathrm{~min}$. The power estimates obtained when $I_{m}$ is incorporated (in this particular example $w_{1}=w_{2}$ ) approach the actual load evolution better than in absence of $I_{m}$ (stepwise solid gray line).

The case in which both loads evolve in opposite directions is shown in Fig. 4. In this case, note that, since the total aggregated load is decreasing, the addition of $I_{m}$ is beneficial for the load which is actually decreasing, $P_{2}$, but detrimental to $P_{1}$, whose increasing trend remains hidden (unobservable) when both loads are sensed upstream. From the point of view of the losing load, $P_{1}$, it would be preferable not to include $I_{m}$ in the model, since the persistent pseudomeasurement value (stepwise solid gray line) would better approximate the actual load evolution.

This somewhat counterintuitive conclusion (i.e., adding an accurate measurement can be counterproductive in certain cases) will be reaffirmed by the results presented below. Needless to mention, such limitations vanish when sufficient redundancy levels are achieved.

\section{SOLUTION ENHANCEMENTS}

So far it has been implicitly assumed that pseudomeasurements are only updated every $n$ measurement snapshots. In other words, at time instant $t_{k}$, the TSSE combines the current measurement snapshot, $z_{m, k}$, with a pseudomeasurement value, $z_{p, k}$, given by the latest available pseudomeasurement

$$
z_{p, k}=z_{p, j} \quad k=j, \ldots, j+n-1 .
$$

This implies that pseudomeasurements are assumed to evolve in a stepwise fashion, as suggested by the uppermost diagram of Fig. 5. 


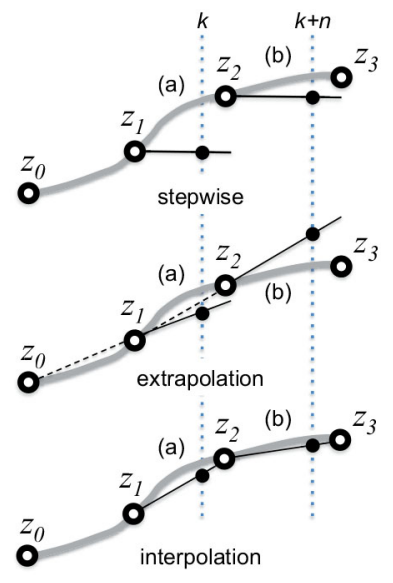

Fig. 5. Generation of pseudomeasurement intermediate values.

Depending on whether future pseudomeasurement values are available in advance or not, other strategies to generate intermediate pseudomeasurement values are possible, as discussed below.

\section{A. Pseudomeasurement Extrapolations}

The accuracy of the estimates can be frequently improved if, instead of keeping the components of $z_{p}$ constant since the last update (stepwise evolution), their values are obtained by linear extrapolation from the last two samples (higher-order extrapolation is also possible, but the results are usually worse owing to longer transient periods). Mathematically

$$
z_{p, k}=z_{p, j}+\frac{t_{k}-t_{j}}{T_{p}}\left(z_{p, j}-z_{p, j-1}\right) \quad k=j, \ldots, j+n-1 .
$$

The middle diagram of Fig. 5 shows two consecutive intervals in which linear extrapolation behaves differently. At interval (a) the linearly extrapolated value approximates the actual evolution of the pseudo-measured quantity better than the latest available value, $z_{1}$. At interval (b), however, owing to the sudden change of slope, linearly extrapolating the pseudomeasurement is worse than just keeping the previous value, $z_{2}$. As shown in Section VI, linear extrapolation is helpful in a majority of cases to improve the estimation provided otherwise by the vanilla stepwise evolution. This happens when the time constants characterizing the load evolution are large enough compared with the refreshing rate of pseudomeasurements.

\section{B. Pseudomeasurement Interpolations}

Obtaining intermediate pseudomeasurements by extrapolation is the only choice when future information about the monitored quantity is missing. This is the case, for instance, of some distributed generators, usually burning fossil fuels, whose energy production is not forecasted but rather measured and collected at a relatively slow rate compared to regular SCADA measurements.

In practice, however, future pseudomeasurement values are almost always available, usually with decreasing accuracy as

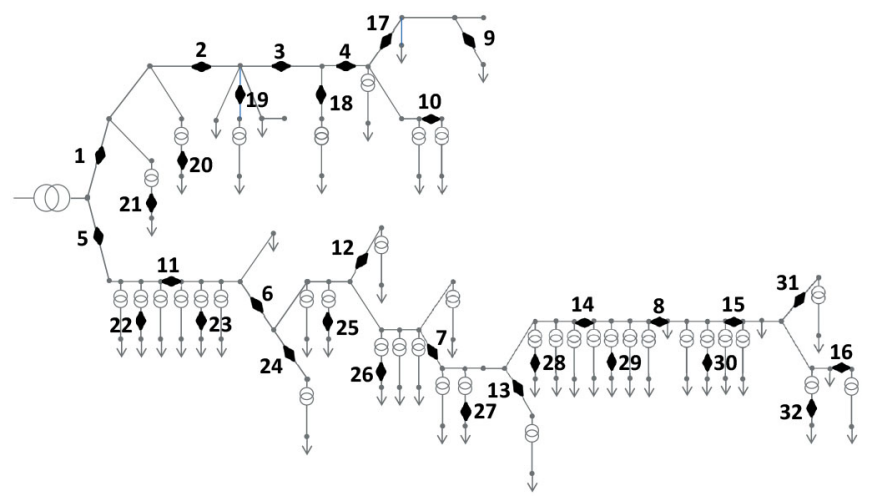

Fig. 6. 100-bus, 15-kV test network.

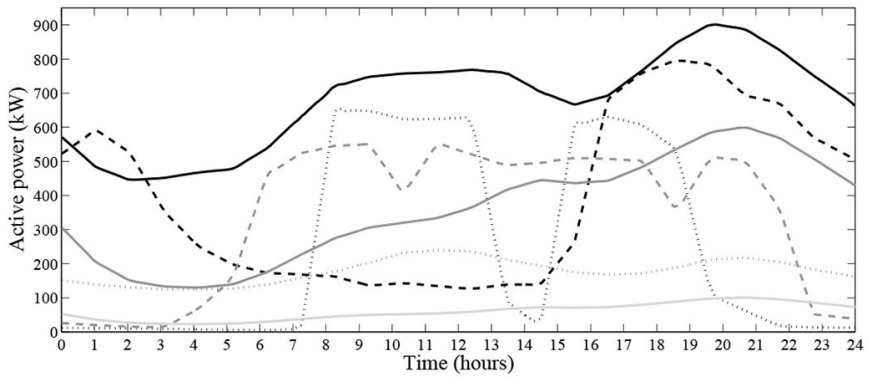

Fig. 7. Examples of 24-h load patterns.

time elapses. For instance, the production of a wind generator for the next hour can be predicted with reasonable accuracy, and the same can be said of a PV farm. On the other hand, loads provided by service transformers can also be forecasted, usually within $\pm 5 \%$ confidence intervals.

In those cases, intermediate values can be easily obtained by linearly interpolating consecutive pseudomeasurements, as shown in the lower diagram of Fig. 5. Mathematically

$$
z_{p, k}=z_{p, j}+\frac{t_{k}-t_{j}}{T_{p}}\left(z_{p, j+1}-z_{p, j}\right) \quad k=j, \ldots, j+n-1
$$

\section{Test Results}

The proposed TSSE model and solution refinements have been tested on a 15-kV, 100-bus distribution network (Fig. 6), made up of two feeders (11.8 and $8.7 \mathrm{~km}$ long) and $38 \mathrm{sec}-$ ondary transformers. This real system delivers energy to a mix of residential, industrial, and commercial loads, comprising a heterogeneous set of load patterns, some of which are shown for illustrative purposes in Fig. 7. Twenty-four-hour active power consumptions are known at all nodes, while reactive powers are obtained by applying typical power factors for each customer type [15]. This leads to the branch power flow profiles shown in decreasing order in Fig. 8. In addition, the head voltage magnitude is assumed to be constant throughout the 24-h period. This allows a load flow to be run, the results of which are considered as exact values for simulation purposes. The resulting voltage magnitudes for the simulation time period are shown in Fig. 9. As expected, voltage magnitudes drop when the power consumption increases.

In order to generate realistic sets of measurements $\left(z_{m}\right)$ and pseudomeasurements $\left(z_{p}\right)$, random errors have been added to 


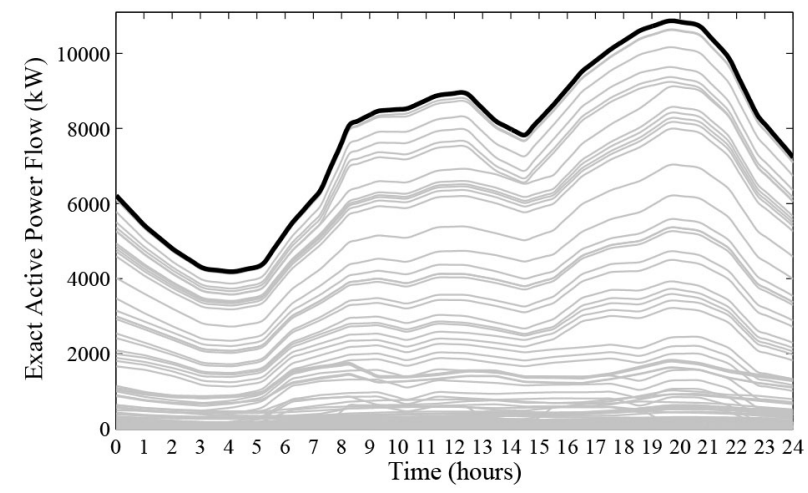

Fig. 8. Branch active power flows in decreasing order.

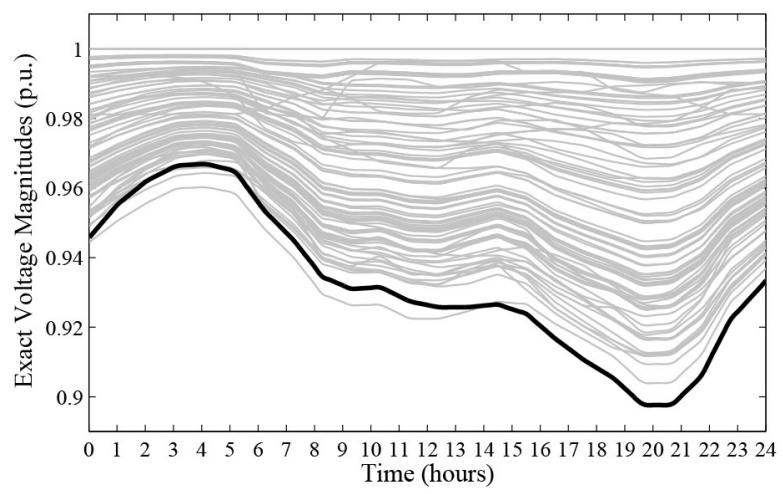

Fig. 9. Bus voltage magnitudes in decreasing order.

the exact 24-h quantities provided by the load flow solution (except for the 38 zero-injection buses corresponding to the $15-\mathrm{kV}$ side of secondary transformers). Each 24-h error pattern is simulated by means of a sinusoidal wave of random amplitude and phase angle, spanning the 24-h period, plus a random DC component, yielding together maximum peak errors of about $10 \%$ for $z_{p}$ and $1 \%$ for $z_{m}$. Snapshots of sets $z_{p}$ and $z_{m}$ are then obtained by sampling the 24-h noisy curves at intervals $T_{p}=15$ minutes and $T_{m}=1$ minute, respectively $(n=15)$. In future smart grids $n$ can be significantly reduced, particularly if smart meter information is processed in a distributed manner, while the number of measurements in $z_{m}$ will steadily increase as distribution automation devices proliferate.

Pseudomeasurements in $z_{p}$ comprise active and reactive power injections at all buses where loads are connected to (zero-injection buses are handled as very accurate, constantly available measurements). In addition to the voltage magnitude at the head bus, fast-rate measurements $\left(z_{m}\right)$ include sets of Ampere measurements, more or less uniformly distributed throughout the feeders. Three scenarios, labeled A, B, and C have been considered, including 8, 16, and 32 current measurements, respectively, placed as shown in Fig. 6. Scenario A includes measurements numbered from 1 to 8 ( 4 of them in each feeder), scenario B measurements 1 to 16 , and scenario $C$ measurements 1 to 32 , leading to really low redundancy levels $(1.04,1.08$, and 1.16 , respectively), in accordance anyway to what can be expected in future smart grids. The base-case scenario, in which only $z_{p}$ and the head bus voltage magnitude are available (i.e., without current measurements), has been also analyzed. This is simply a load flow solution using a critical set of erroneous data (no possibility of filtering errors), which will be useful to quantify the improvements brought about by the incorporation of $z_{m}$ in the different scenarios.

In real life, determining the accuracy of pseudomeasurements is not a trivial task. For this reason, in absence of better alternative criteria, weights adopted for the TSSE model have been set in inverse proportion to the pseudomeasurement and measurement values, and then those of $z_{m}$ are multiplied by 10 to reflect their higher accuracy compared with $z_{p}$. Simulations for the base case and the three barely redundant scenarios have been performed and the results obtained are compared with exact values. Solution enhancements described in Section V (extrapolation and interpolation) have been also tested.

Fig. 10 shows the 24-h evolution of active power at a representative load bus, for both the base case ( $z_{p}$ only) and scenario A (8 branch measurements). Exact values are also provided for comparison. The diagrams on top show the results obtained when $z_{p}$ is kept constant between slow-rate snapshots (stepwise evolution assumed). In average, estimates obtained in scenario A are better than those of the base case, particularly at the central hours. The zoomed image on the right, corresponding to the interval 13:45-16:15 h, shows the stepwise, and sometimes sawtooth shape evolution of estimates, according to the behavior theoretically predicted in Section IV for very low redundancy levels and loads with opposite trends.

The central curves in Fig. 10 show the results obtained when extrapolation is applied to update future $z_{p}$ values before they are available. In this case, the evolution of estimates is smoother than in the previous one. However, when there is a sudden slope change, the estimator needs a time interval of width $T_{p}$ to get adapted to the new load trend.

The bottom diagrams show the results when interpolation of $z_{p}$ is performed, which is possible only when future values are forecasted or somehow computed in advance. Compared to the two previous cases, this scheme clearly provides the best performance.

Similar conclusions apply to the rest of nodes. At some time instants, the counterintuitive behavior illustrated in Fig. 4, namely poorer estimates of certain downstream powers originating by the incorporation of a current measurement, can be clearly observed. As an example, let us consider the influence of current measurement \#8, located at the branch between nodes 131 and 132, in the estimates of bus injection $P_{132}$ and the power flow feeding the rest of loads downstream, $P_{132-133}$ (Fig. 11 represents the area of the test network involved in this example). The exact values for $P_{132}$ and $P_{132-133}$, as well as the estimates with and without current measurements (scenario A and base case) are shown in Fig. 12. It can be observed how, for the first $2 \mathrm{~h}$ represented, when both power quantities follow a decreasing trend, the incorporation of current measurement \#8 is beneficial for both magnitudes. However, after hour 3, the power flow $P_{132-133}$ starts increasing. From this time on, particularly after hour 5 when the positive slope of $P_{132-133}$ becomes steeper, the estimate of $P_{132}$ in scenario A starts to deteriorate compared to the initial pseudomeasurement (base case), whereas $P_{132-133}$ continues benefiting 

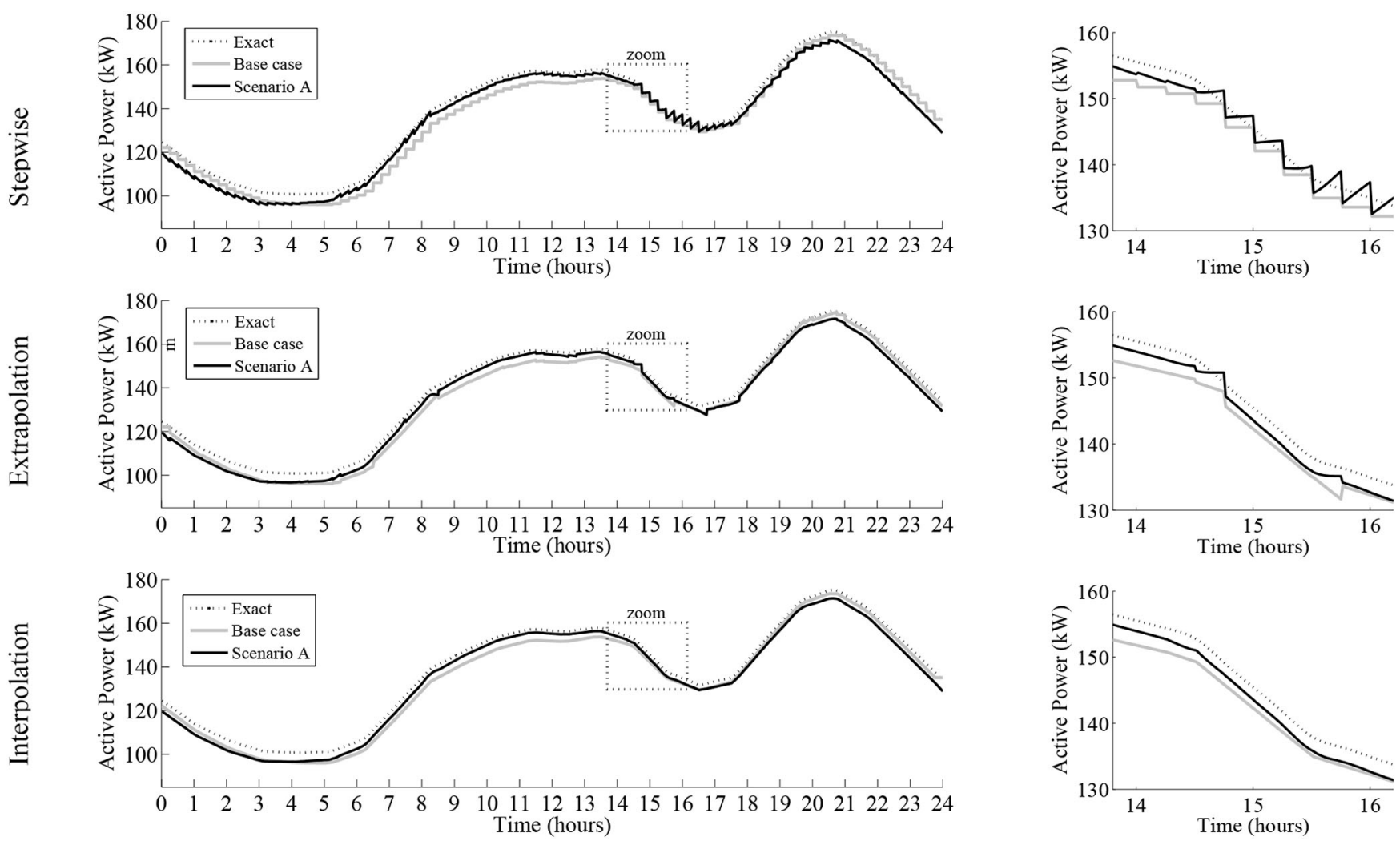

Fig. 10. Evolution of active power estimates at a typical load bus in scenario A, when (top) $z_{p}$ is kept constant between snapshots, (middle) extrapolation is used, and (bottom) interpolation is performed.

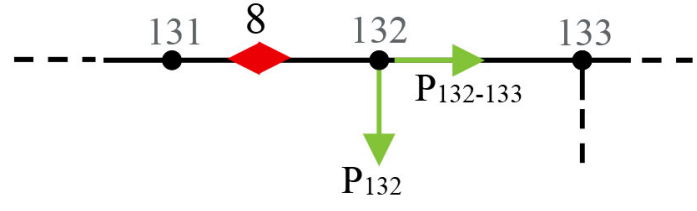

Fig. 11. Zoomed view of the test system around bus 132 .

from the current measurement. It is worth noting that $P_{132-133}$ is around five times larger than $P_{132}$ and, therefore, even though $P_{132}$ estimates worsen, the average estimation errors get reduced by the addition of measurement \#8, as expected.

The accuracy of estimates in scenarios A-C has been numerically compared with that of the base case. Average absolute values of errors (estimated minus exact) of power flow, power injection and voltage magnitude estimates (extended to all branches or nodes, respectively, for every minute in $24 \mathrm{~h}$ ), are presented in Table I. As expected, the average absolute errors decrease when the number of current measurements increases, but the larger relative improvement with respect to the base case is obtained with scenario A, comprising just eight current measurements. The adoption of extrapolation and interpolation strategies significantly improves the estimates and can even compensate for the lack of measurements. For instance, using interpolation in scenario A (eight measurements) provides comparable or better results than the vanilla stepwise strategy in scenario C (32 measurements).

In addition to absolute errors, Table I also shows, for power injection pseudomeasurements $\left(z_{p}\right)$, the average error reduction (in \%) of the estimated values compared to the initial errors associated to the noisy values (base case, stepwise evolution), rather than exact values. Error reductions for active power injections ranging from $11.3 \%$ (scenario A, stepwise) to $45.5 \%$ (scenario $\mathrm{C}$ with interpolation) have been obtained. It is worth noting that for the base case (no current measurements at all) simply extrapolating and interpolating the pseudomeasurements reduces the original errors by $18.4 \%$ and $22.8 \%$, respectively. Similar conclusions apply to reactive power injections.

Since adding more real-time measurements has a significant associated cost, each user of the TSSE should determine at the planning stage how many extra measurements are required to achieve the desired accuracy, for given sampling rates, pseudomeasurement quality and diversity of load evolution. If all loads have very homogeneous trends, as happens for instance when the feeders cover a purely residential area, then perhaps one or two Ampere measurements (at the head and middle of the feeder), might suffice to complement forecasted load values (in the limit this reduces to a simple load allocation scheme). However, as happens in the distribution system tested herein, if there is a mix of load types, some of them raising when others are decreasing, then it is not so obvious what is the optimal number and placement of realtime measurements (this constitutes an interesting optimization problem).

The improvement brought about by the addition of eight current measurements (scenario A) is better visualized in Fig. 13, representing the 24-h evolution of active power 


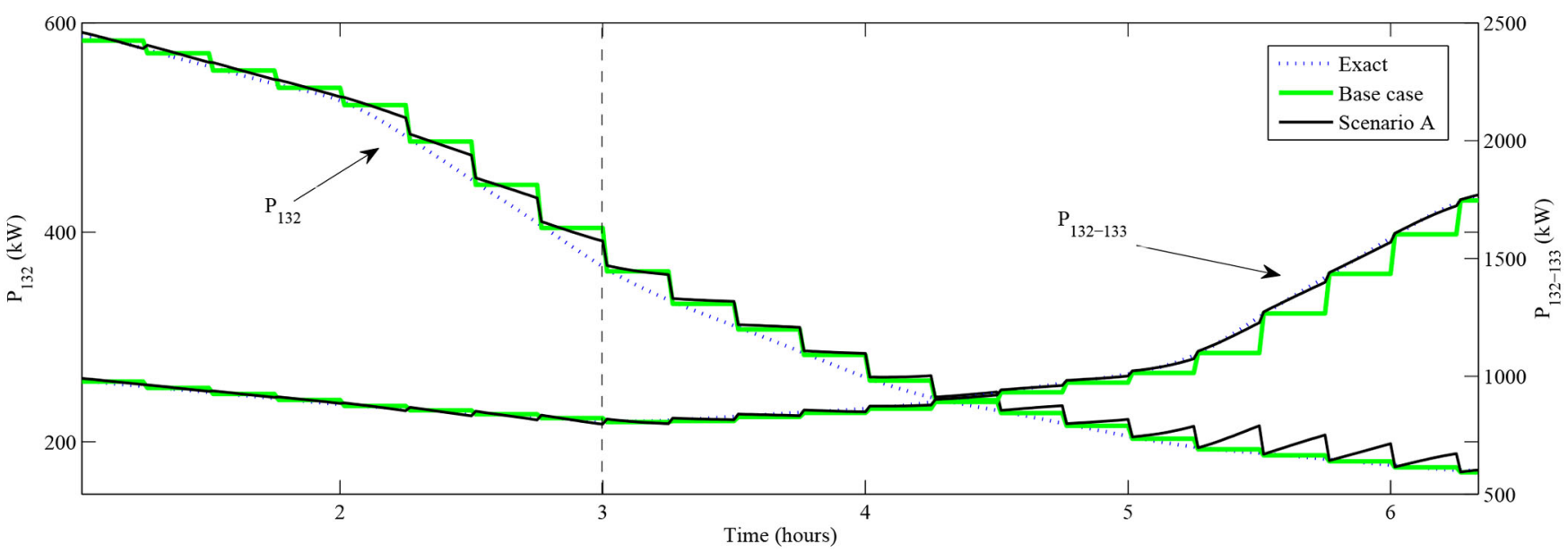

Fig. 12. Illustration of active power estimates at time intervals with homogeneous and opposite load trends (stepwise scheme).

TABLE I

Average Absolute Errors and Relative Error Reductions (\%) Compared to Pseudomeasured Values

\begin{tabular}{|c|c|c|c|c|c|c|c|c|c|c|c|c|c|}
\hline & & \multicolumn{4}{|c|}{ Stepwise } & \multicolumn{4}{|c|}{ Extrapolation } & \multicolumn{4}{|c|}{ Interpolation } \\
\hline \multicolumn{2}{|c|}{ Magnitudes Scenarios } & Base case & A & B & $\mathrm{C}$ & Base case & $\mathrm{A}$ & B & $\mathrm{C}$ & Base case & A & B & $\mathrm{C}$ \\
\hline$P_{i j}$ & $(\mathrm{~kW})$ & 19.74 & 6.05 & 5.25 & 4.60 & 9.97 & 5.12 & 4.69 & 4.09 & 8.34 & 4.80 & 4.45 & 3.86 \\
\hline$Q_{i j}$ & (kvar) & 9.57 & 5.20 & 5.08 & 4.91 & 4.88 & 3.39 & 3.13 & 2.99 & 4.47 & 3.28 & 3.01 & 2.85 \\
\hline$V_{i}$ & (p.u.) $* 10^{-3}$ & 0.38 & 0.12 & 0.11 & 0.09 & 0.18 & 0.11 & 0.10 & 0.08 & 0.16 & 0.11 & 0.10 & 0.08 \\
\hline \multirow{2}{*}{$P_{i}$} & $(\mathrm{~kW})$ & 4.98 & 4.42 & $\overline{4.16}$ & 3.32 & 4.06 & 3.83 & 3.68 & 2.89 & 3.84 & 3.62 & 3.49 & 2.71 \\
\hline & $(\%)$ & - & 11.3 & 16.4 & 33.3 & 18.4 & 23.0 & 26.0 & 41.9 & 22.8 & 27.2 & 29.9 & 45.5 \\
\hline \multirow{2}{*}{$Q_{i}$} & (kvar) & 2.10 & 1.88 & 1.82 & 1.63 & 1.75 & 1.66 & 1.61 & 1.42 & 1.69 & 1.61 & 1.55 & 1.37 \\
\hline & $(\%)$ & - & 10.3 & 13.0 & 22.4 & 16.8 & 20.8 & 23.3 & 32.1 & 19.6 & 23.3 & 26.0 & 34.7 \\
\hline
\end{tabular}

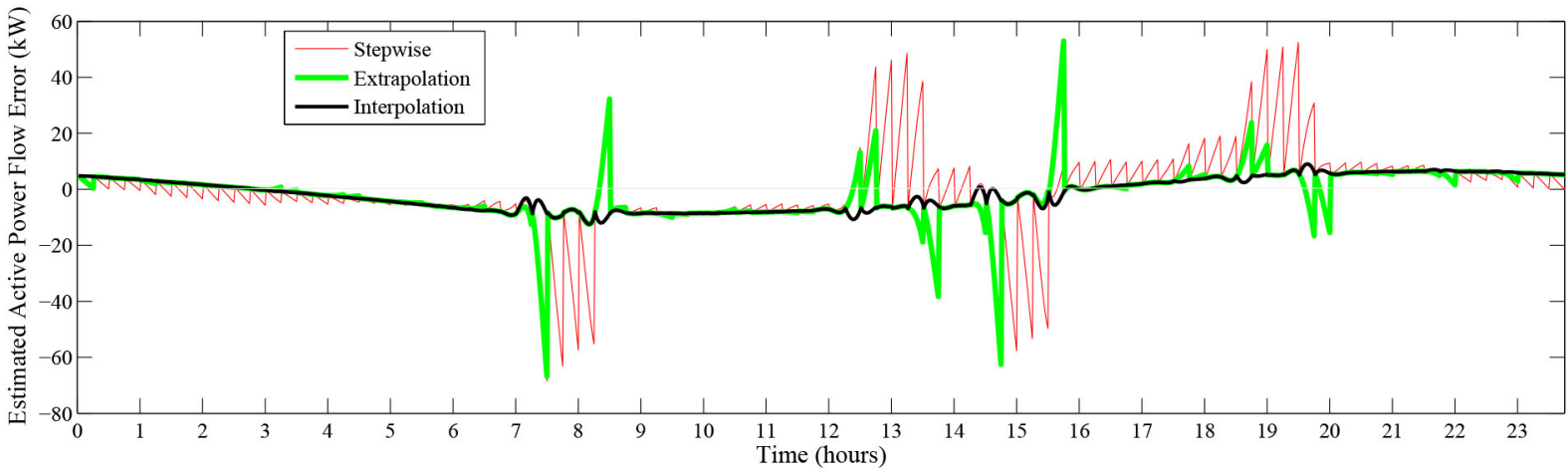

Fig. 13. Evolution of active power flow errors for the branch with largest load (scenario A).

flow errors corresponding to the branch section carrying the largest apparent power (shown in boldface in Fig. 8). Both positive and negative errors approaching $60 \mathrm{~kW}$ can be seen for the stepwise and extrapolation approaches, the number of peaks being significantly reduced in the latter case. It is worth pointing out that, as theoretically predicted, error peaks take place when there are sudden slope changes in the load evolution, which happens approximately at around 7:30-8:30, 12:00-16:00, and 18:30-20:00 $\mathrm{h}$ (the reader is referred to the quickly changing load patterns of Fig. 7). The interpolation approach virtually gets rid of the oscillations, keeping the maximum errors within $\pm 10 \mathrm{~kW}$ for a branch carrying an average load of $8000 \mathrm{~kW}$.

Fig. 14 shows similar results for the evolution of voltage magnitude errors corresponding to the node with largest voltage drop (shown in boldface in Fig. 9). Peak errors of -0.0013 p.u. (about $20 \mathrm{~V}$ on a $15 \mathrm{kV}$ basis) are reached by stepwise and extrapolation approaches, while using interpolation the voltage errors do not exceed -0.0005 p.u. $(7.5 \mathrm{~V})$.

Similar results are obtained for the rest of nodes and branches. For the rest of scenarios, similar trends are observed, 


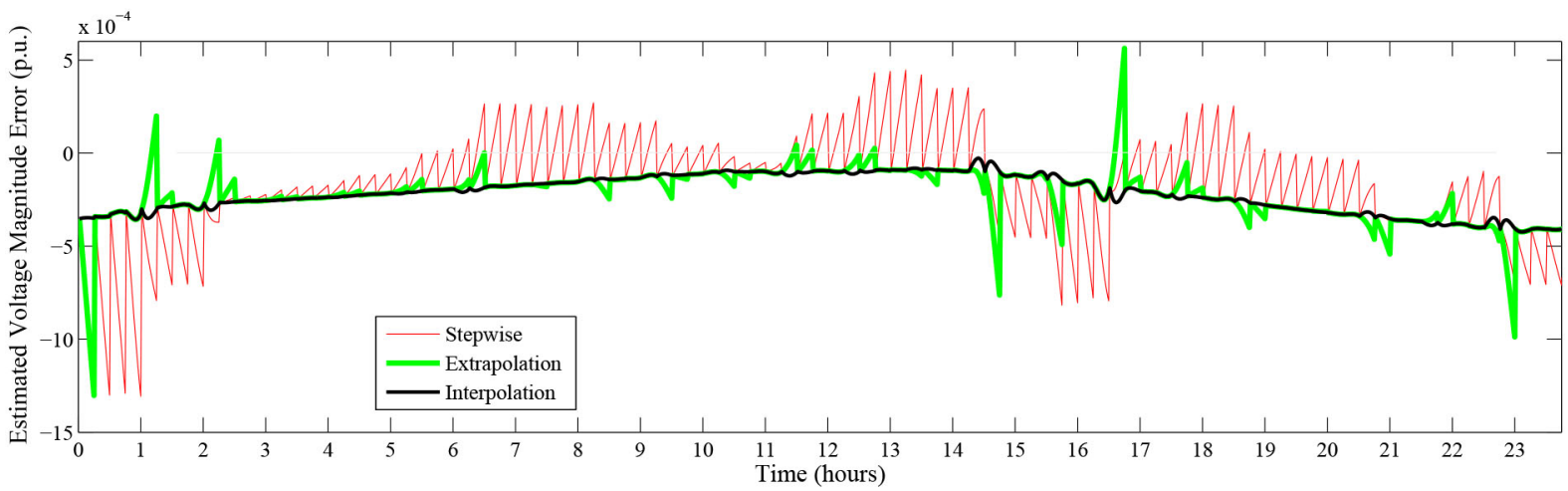

Fig. 14. Evolution of voltage magnitude errors for the node with largest voltage drop (scenario A).

but the average errors decrease with the incorporation of current measurements.

\section{CONCLUSION}

Based on an analysis (types, latencies, and accuracy) of the information sources available at the distribution level, a state estimator in two time scales is proposed in this paper. It integrates a critical set of pseudomeasurements with very few redundant measurements, more accurate and captured $n$ times faster. Limitations of the proposed model arising in low-redundancy environments, particularly when heterogenous load patterns coexist in the same feeder, are discussed, and several enhancements to deal with pseudomeasurement obsolescence are proposed.

Test results on a real distribution system, feeding a diversity of load patterns, fully confirms the suitability of the TSSE to both improve the accuracy and increase the latency of the load flow solutions that could otherwise be computed if only pseudomeasurements were used. In the mid term, forecasted pseudomeasurements will be gradually replaced by smart meter readings and the number of measurements will steadily increase, but the need to handle two time scales will persist.

\section{APPENDIX}

In the simple networks of Fig. 2, the measurement model comprises two pairs of power pseudomeasurements

$$
\left.\begin{array}{rl}
P_{1}^{m} & =P_{1}+\epsilon_{P 1} \\
P_{2}^{m} & =P_{2}+\epsilon_{P 2} \\
Q_{1}^{m} & =Q_{1}+\epsilon_{Q 1} \\
Q_{2}^{m} & =Q_{2}+\epsilon_{Q 2}
\end{array}\right\} \rightarrow z_{1}=x+\epsilon
$$

plus an Ampere measurement which, being much more accurate than the set of power pseudomeasurements, can be considered for our purposes as an equality constraint. Ignoring branch losses, with $V_{i}=1$, it can be approximately expressed as follows:

$$
I_{m}^{2}=\left(P_{1}+P_{2}\right)^{2}+\left(Q_{1}+Q_{2}\right)^{2} \quad \rightarrow \quad z_{2}=s(x) .
$$

In compact form, the objective function associated with the equality-constrained WLS SE can be written as

$$
\mathcal{L}=\frac{1}{2}\left(z_{1}-x\right)^{T} W\left(z_{1}-x\right)-\lambda^{T}\left[z_{2}-s(x)\right]
$$

with $x$ given by

$$
x=\left[P_{1}, P_{2}, Q_{1}, Q_{2}\right]^{T} .
$$

The estimate, $\hat{x}$, is the one satisfying the first-order optimality conditions

$$
\begin{array}{r}
W\left(z_{1}-\hat{x}\right)-S^{T} \lambda=0 \\
z_{2}-s(\hat{x})=0
\end{array}
$$

where $S$ is the Jacobian of $s(x)$.

Assuming the weighting coefficients of $P$ and $Q$ are the same, (12) can be rewritten as

$$
\begin{aligned}
& \hat{P}_{1}=P_{1}^{m}-2 w_{1}^{-1}\left(\hat{P}_{1}+\hat{P}_{2}\right) \lambda \\
& \hat{P}_{2}=P_{2}^{m}-2 w_{2}^{-1}\left(\hat{P}_{1}+\hat{P}_{2}\right) \lambda \\
& \hat{Q}_{1}=Q_{1}^{m}-2 w_{1}^{-1}\left(\hat{Q}_{1}+\hat{Q}_{2}\right) \lambda \\
& \hat{Q}_{2}=Q_{2}^{m}-2 w_{2}^{-1}\left(\hat{Q}_{1}+\hat{Q}_{2}\right) \lambda .
\end{aligned}
$$

Adding (14) to (15) and (16) to (17) yields

$$
\begin{aligned}
\hat{P}_{1}+\hat{P}_{2} & =\frac{\left(P_{1}^{m}+P_{2}^{m}\right)}{1+2 \lambda\left(w_{1}^{-1}+w_{2}^{-1}\right)} \\
\hat{Q}_{1}+\hat{Q}_{2} & =\frac{\left(Q_{1}^{m}+Q_{2}^{m}\right)}{1+2 \lambda\left(w_{1}^{-1}+w_{2}^{-1}\right)} .
\end{aligned}
$$

Substituting (18) and (19) into (9)

$$
I_{m}^{2}=\frac{\left(P_{1}^{m}+P_{2}^{m}\right)^{2}+\left(Q_{1}^{m}+Q_{2}^{m}\right)^{2}}{\left[1+2 \lambda\left(w_{1}^{-1}+w_{2}^{-1}\right)\right]^{2}} .
$$

Let us define $K_{m}$ as

$$
K_{m}=\frac{1}{1+2 \lambda\left(w_{1}^{-1}+w_{2}^{-1}\right)} .
$$

Then

$$
K_{m}^{2}=\frac{I_{m}^{2}}{\left(P_{1}^{m}+P_{2}^{m}\right)^{2}+\left(Q_{1}^{m}+Q_{2}^{m}\right)^{2}}
$$

which allows rewriting (18) and (19) as

$$
\begin{aligned}
& \hat{P}_{1}+\hat{P}_{2}=K_{m}\left(P_{1}^{m}+P_{2}^{m}\right) \\
& \hat{Q}_{1}+\hat{Q}_{2}=K_{m}\left(Q_{1}^{m}+Q_{2}^{m}\right) .
\end{aligned}
$$


Going back (14) and (15), they can be written in matrix form as (same for $\hat{Q}_{i}$ and $Q_{i}^{m}$ )

$$
\left[\begin{array}{cc}
w_{1}+2 \lambda & 2 \lambda \\
2 \lambda & w_{2}+2 \lambda
\end{array}\right]\left[\begin{array}{l}
\hat{P}_{1} \\
\hat{P}_{2}
\end{array}\right]=\left[\begin{array}{l}
w_{1} P_{1}^{m} \\
w_{2} P_{2}^{m}
\end{array}\right]
$$

and, explicitly computing the inverse

$$
\left[\begin{array}{l}
\hat{P}_{1} \\
\hat{P}_{2}
\end{array}\right]=\frac{1}{w_{1}+w_{2}}\left[\begin{array}{ll}
w_{1}+w_{2} K_{m} & \left(K_{m}-1\right) w_{2} \\
\left(K_{m}-1\right) w_{1} & w_{2}+w_{1} K_{m}
\end{array}\right]\left[\begin{array}{c}
P_{1}^{m} \\
P_{2}^{m}
\end{array}\right]
$$

In incremental form, expressions (4) result.

\section{REFERENCES}

[1] F. C. Schweppe, J. Wildes, and D. B. Rom, "Power system static state estimation, part I, II, III," IEEE Trans. Power App. Syst., vol. PAS-89, no. 1, pp. 120-135, Jan. 1970.

[2] I. Roytelman and S. M. Shahidehpour, "State estimation for electric power distribution systems in quasi real-time conditions," IEEE Trans. Power Del., vol. 8, no. 4, pp. 2009-2015, Oct. 1993.

[3] C. N. Lu, J. H. Teng, and W.-H. E. Liu, "Distribution state estimation," IEEE Trans. Power Syst., vol. 10, no. 1, pp. 229-240, Feb. 1995.

[4] R. Hoffman, S. Lefebvre, and J. Prevost, "Distribution state estimation: A fundamental requirement for the smart grid," in DistribuTech, Tampa, FL, USA, Mar. 2010.

[5] I. Dzafic, S. Henselmeyer, and H. T. Neisius, "Real-time distribution system state estimation," in Proc. Conf. IPEC, Singapore, Oct. 2010, pp. 22-27.

[6] I. Dzafic, M. Gilles, R. A. Jabr, B. C. Pal, and S. Henselmeyer, "Real time estimation of loads in radial and unsymmetrical threephase distribution networks," IEEE Trans. Power Syst., vol. 28, no. 4, pp. 4839-4848, Nov. 2013.

[7] A. Bose, "Smart transmission grid applications and their supporting infrastructure," IEEE Trans. Smart Grid, vol. 1, no. 1, pp. 11-19, Jun. 2010.

[8] S. T. Mak and N. Farah, "Synchronizing SCADA and smart meters operation for advanced smart distribution grid applications," in Proc. IEEE PES Innov. Smart Grid Technol., Washington, DC, USA, pp. 1, 7 2012.

[9] A. Gómez-Expósito, A. Abur, A. de la Villa Jaén, and C. Gómez-Quiles, "A multilevel state estimation paradigm for smart grids," Proc. IEEE, vol. 99, no. 6, pp. 952-976, Jun. 2011.

[10] C. Gómez-Quiles, A. Gómez-Expósito, and A. de la Villa Jaén, "State estimation for smart distribution substations," IEEE Trans. Smart Grid, vol. 3, no. 2, pp. 986-995, Jun. 2012.

[11] K. Samarakoon, J. Wu, J. Ekanayake, and N. Jenkins, "Use of delayed smart meter measurements for distribution state estimation," in Proc. IEEE Power Energy Soc. Gen. Meet., San Diego, CA, USA, Jul. 2011, pp. 1,6 .

[12] J. Wu, Y. He, and N. Jenkins, "A robust state estimator for medium voltage distribution networks," IEEE Trans. Power Syst., vol. 28, no. 2, pp. 1008-1016, May 2013.

[13] A. Abdel-Majeed, S. Tenbohlen, D. Schollhorn, and M. Braun, "Development of state estimator for low voltage networks using smart meters measurement data," in Proc. Grenoble PowerTech, Grenoble, France, pp. 1, 6, 2013.

[14] X. Feng, F. Yang, and W. Peterson, "A practical multi-phase distribution state estimation solution incorporating smart meter and sensor data," in Proc. IEEE Power Energy Soc. (PES) Gen. Meet., San Diego, CA, USA, 2012, pp. $1,6$.
[15] C. Carmona, E. Romero-Ramos, and J. Riquelme, "Fast and reliable distribution load and state estimator," Electron. Power Syst. Res., vol. 101, pp. 110-124, Aug. 2013.

[16] J. Chow and P. V. Kokotovic, "Two-time-scale feedback design of a class of nonlinear systems," IEEE Trans. Autom. Control, vol. 23, no. 3, pp. 438-443, Jun. 1978.

[17] K. Khorasani and M. A. Pai, "Two time scale decomposition and stability analysis of power systems," IEE Proc. D, Control Theory Appl., vol. 135, no. 3, pp. 205-212, May 1988.

[18] H. Hofmann and S. R. Sanders, "Speed-sensorless vector torque control of induction machines using a two-time-scale approach," IEEE Trans. Ind. Appl., vol. 34, no. 1, pp. 169-177, Jan./Feb. 1998.

[19] G. Yin, Z. Qing, J. B. Moore, and J. L. Yuan, "Continuous-time tracking algorithms involving two-time-scale Markov chains," IEEE Trans. Signal Process., vol. 53, no. 12, pp. 4442-4452, Dec. 2005.

[20] A. M. Nagy-Kiss, B. Marx, G. Mourot, G. Schutz, and J. Ragot, "State estimation of two-time scale multiple models with unmeasurable premise variables. Application to biological reactors," in Proc. 49th IEEE Conf. Decis. Control (CDC), Atlanta, GA, USA, Dec. 2010, pp. 5689-5694.

[21] R. Singh, B. C. Pal, and R. A. Jabr, "Choice of estimator for distribution system state estimation," IET Gener. Transmiss. Distrib., vol. 3, no. 7, pp. 666-678, 2009.

[22] M. Glavic and T. Van Cutsem, "Reconstructing and tracking network state from a limited number of synchrophasor measurements," IEEE Trans. Power Syst., vol. 28, no. 2, pp. 1921-1929, May 2013.

[23] P. Kansal and A. Bose, "Bandwidth and latency requirements for smart transmission grid applications," IEEE Trans. Smart Grid, vol. 3, no. 3, pp. 1344-1351, Sep. 2012.

Antonio Gómez-Expósito (F’05) received the Industrial Engineering degree in electrical engineering and the Doctor Engineering degree in power engineering from the University of Seville, Seville, Spain, in 1982 and 1985, respectively.

$\mathrm{He}$ is currently the Endesa Red Industrial Chair Professor with the University of Seville. His research interests include optimal power system operation, state estimation, digital signal processing, and control of flexible ac transmission system devices.

Catalina Gómez-Quiles (M'12) received the Engineering degree in electrical engineering from the University of Seville, Seville, Spain; the M.Sc. degree in electrical engineering from McGill University, Montreal, QC, Canada, in 2009; and the Doctor Engineering degree in power engineering from the University of Seville, in 2012.

Her research interests include mathematical and computer models for power system analysis.

Izudin Džafić (SM'13) received the Ph.D. degree in electrical engineering from the University of Zagreb, Zagreb, Croatia, in 2002.

He is currently with Siemens AG, Nuremberg, Germany, where he is the Head of the department and the Chief Product Owner for Distribution Network Analysis Research and Development. His research interests include power system modeling, development, and application of fast computing to power systems simulations.

Dr. Džafić is a member of the IEEE Power and Energy Society and the IEEE Computer Society. 